\title{
The methods for variational inequality problems and fixed point of $\kappa$-strictly pseudononspreading mapping
}

\section{Atid Kangtunyakarn*}

\section{"Correspondence:}

beawrock@hotmail.com

Department of Mathematics,

Faculty of Science, King Mongkut's

Institute of Technology Ladkrabang,

Bangkok 10520, Thailand

\begin{abstract}
In this paper, we introduce the methods for finding a common element of the set of fixed points of a $\boldsymbol{\kappa}$-strictly pseudononspreading mapping and a finite family of the set of solutions of variational inequality problems. The strong convergence theorem of the proposed method is established under some suitable control conditions. Moreover, by using our main result, we prove interesting theorem involving an iterative scheme for finding a common element of the set of fixed points of a $\kappa$-strictly pseudononspreading mapping and a finite family of the set of fixed points of a $\boldsymbol{\kappa}_{i}$-strictly pseudocontractive mappings.
\end{abstract}

Keywords: variational inequality problems; $\kappa$-strictly pseudononspreading mapping; strictly pseudocontractive mapping

\section{Introduction}

Let $C$ be a nonempty closed convex subset of a real Hilbert space $H$. Recall that the mapping $T: C \rightarrow C$ is said to be nonexpansive if $\|T x-T y\| \leq\|x-y\|$ for all $x, y \in C$. In 2008, Kohsaka and Takahashi [1] introduced the nonspreading mapping in Hilbert spaces $H$ which is defined as follows: $2\|T x-T y\|^{2} \leq\|T x-y\|^{2}+\|x-T y\|^{2}, \forall x, y \in C$. Following the terminology of Browder and Petryshyn [2], in 2011, Osilike and Isiogugu [3] introduced that the mapping $T: C \rightarrow C$ is called a $\kappa$-strictly pseudononspreading mapping if there exists $\kappa \in[0,1)$ such that

$$
\|T x-T y\|^{2} \leq\|x-y\|^{2}+\kappa\|(I-T) x-(I-T) y\|^{2}+2\langle x-T x, y-T y\rangle
$$

for all $x, y \in C$. Clearly every nonspreading mapping is $\kappa$-strictly pseudononspreading; see, for example, [3]. A point $x \in C$ is called a fixed point of $T$ if $T x=x$. The set of fixed points of $T$ is denoted by $F(T)=\{x \in C: T x=x\}$.

Let $A: C \rightarrow H$. The variational inequality problem is to find a point $u \in C$ such that

$$
\langle A u, v-u\rangle \geq 0
$$

for all $v \in C$. The set of solutions of (1.1) is denoted by $\operatorname{VI}(C, A)$.

The variational inequality has emerged as a fascinating and interesting branch of mathematical and engineering sciences with a wide range of applications in industry, finance, economics, social, ecology, regional, pure and applied sciences; see, e.g., [4-7].

(c) 2013 Kangtunyakarn; licensee Springer. This is an Open Access article distributed under the terms of the Creative Commons Attribution License (http://creativecommons.org/licenses/by/2.0), which permits unrestricted use, distribution, and reproduction in any medium, provided the original work is properly cited. 
A mapping $A$ of $C$ into $H$ is called $\alpha$-inverse strongly monotone (see [8]) if there exists a positive real number $\alpha$ such that

$$
\langle x-y, A x-A y\rangle \geq \alpha\|A x-A y\|^{2}
$$

for all $x, y \in C$.

In 2003, Takahashi and Toyoda [9] proved a convergence theorem for finding a common element of the set of fixed points of nonexpansive mappings and the set of solutions of variational inequalities for $\alpha$-inverse strongly monotone mappings as follows.

Theorem 1.1 Let $K$ be a closed convex subset of a real Hilbert space H. Let $\alpha>0$. Let $A$ be an $\alpha$-inverse strongly monotone mapping of $K$ into $H$, and let $S$ be a nonexpansive mapping of $K$ into itself such that $F(S) \cap V I(K, A) \neq \emptyset$. Let $\left\{x_{n}\right\}$ be a sequence generated by $x_{0} \in K$ and

$$
x_{n+1}=\alpha_{n} x_{n}+\left(1-\alpha_{n}\right) S P_{C}\left(I-\lambda_{n} A\right) x_{n}
$$

for every $n=0,1,2, \ldots$, where $\left\{\lambda_{n}\right\} \subset[a, b]$ for some $a, b \in(0,2 \alpha)$ and $\left\{\alpha_{n}\right\} \subset[c, d]$ for some $c, d \in(0,1)$. Then $\left\{x_{n}\right\}$ converges weakly to $z=F(S) \cap V I(K, A)$, where $z=$ $\lim _{n \rightarrow \infty} P_{F(S) \cap V I(K, A)} x_{n}$.

Recently, Osilike and Isiogugu [3] proved strong convergence theorems for strictly pseudononspreading mappings as follows.

Theorem 1.2 Let $C$ be a nonempty closed convex subset of a real Hilbert space and let $T: C \rightarrow C$ be a $\kappa$-strictly pseudononspreading mapping with a nonempty fixed point set $F(T)$. Let $\beta \in[k, 1)$ and let $\left\{\alpha_{n}\right\}_{n=1}^{\infty}$ be a real sequence in $[0,1)$ such that $\lim _{n \rightarrow \infty} \alpha_{n}=0$ and $\sum_{n=1}^{\infty} \alpha_{n}=\infty$. Let $u \in C$ and let $\left\{x_{n}\right\}_{n=1}^{\infty}$ and $\left\{z_{n}\right\}_{n=1}^{\infty}$ be sequences in $C$ generated from an arbitrary $x_{1} \in C$ by

$$
\left\{\begin{array}{l}
x_{n+1}=\alpha_{n} u+\left(1-\alpha_{n}\right) z_{n}, \quad \forall n \geq 1, \\
z_{n}=\frac{1}{n} \sum_{k=0}^{n-1} T_{\beta}^{k} x_{n}, \quad \forall n \geq 1,
\end{array}\right.
$$

where $T_{\beta}=\beta I+(1-\beta) T$. Then $\left\{x_{n}\right\}_{n=1}^{\infty}$ and $\left\{z_{n}\right\}_{n=1}^{\infty}$ converge strongly to $P_{F(T)} u$, where $P_{F(T)}$ : $H \rightarrow F(T)$ is the metric projection of $H$ onto $F(T)$.

Theorem 1.3 Let $C$ be a nonempty closed convex subset of a real Hilbert space and let $T$ : $C \rightarrow C$ be a $\kappa$-strictly pseudononspreading mapping with a nonempty fixed point set $F(T)$. Let $\beta \in[k, 1)$ and let $T_{\beta}=\beta I+(1-\beta) T$. Let $\left\{\alpha_{n}\right\}_{n=1}^{\infty}$ be a real sequence in $[0,1)$ satisfying the conditions

(C1) $\lim _{n \rightarrow \infty} \alpha_{n}=0$ and

(C2) $\sum_{n=1}^{\infty} \alpha_{n}=\infty$.

Let $u \in C$ be a fixed anchor in $C$ and let $\left\{x_{n}\right\}_{n=1}^{\infty}$ be a sequence in $C$ generated from an arbitrary $x_{1} \in C$ by

$$
x_{n+1}=\alpha_{n} u+\left(1-\alpha_{n}\right) T_{\beta} x_{n}, \quad n \geq 1 .
$$

Then $\left\{x_{n}\right\}_{n=1}^{\infty}$ converges strongly to a fixed point $p$ of $T$. 
Inspired and motivated by [3] and the research in the same direction, we prove a strong convergence theorem of $\kappa$-strictly pseudononspreading mappings and introduce the methods for finding a common element of the set of fixed points of a $\kappa$-strictly pseudononspreading mapping and a finite family of the set of solutions of variational inequality problems. Moreover, by using our main result, we prove an interesting theorem involving an iterative scheme for finding a common element of the set of fixed points of $\kappa$-strictly pseudononspreading mappings and a finite family of the set of fixed points of $\kappa_{i}$-strictly pseudocontractive mappings.

\section{Preliminaries}

We need the following lemmas to prove our main result. Let $H$ be a real Hilbert space and let $C$ be a nonempty closed convex subset of $H$, let $P_{C}$ be the metric projection of $H$ onto $C$, i.e., for $x \in H, P_{C} x$ satisfies the property

$$
\left\|x-P_{C} x\right\|=\min _{y \in C}\|x-y\| .
$$

The following characterizes the projection $P_{C}$.

Lemma 2.1 (See [10]) Given $x \in H$ and $y \in C$. Then $P_{C} x=y$ if and only if the following inequality holds:

$$
\langle x-y, y-z\rangle \geq 0, \quad \forall z \in C
$$

Lemma 2.2 (See [10]) Let $H$ be a Hilbert space, let $C$ be a nonempty closed convex subset of $H$ and let $A$ be a mapping of $C$ into $H$. Let $u \in C$. Then, for $\lambda>0$,

$$
u=P_{C}(I-\lambda A) u \quad \Leftrightarrow \quad u \in V I(C, A),
$$

where $P_{C}$ is the metric projection of $H$ onto $C$.

Lemma 2.3 (See [11]) Let $\left\{s_{n}\right\}$ be a sequence of nonnegative real numbers satisfying

$$
s_{n+1}=\left(1-\alpha_{n}\right) s_{n}+\delta_{n}, \quad \forall n \geq 0,
$$

where $\left\{\alpha_{n}\right\}$ is a sequence in $(0,1)$ and $\left\{\delta_{n}\right\}$ is a sequence such that

(1) $\sum_{n=1}^{\infty} \alpha_{n}=\infty$,

(2) $\lim \sup _{n \rightarrow \infty} \frac{\delta_{n}}{\alpha_{n}} \leq 0$ or $\sum_{n=1}^{\infty}\left|\delta_{n}\right|<\infty$.

Then $\lim _{n \rightarrow \infty} s_{n}=0$.

Lemma 2.4 (See [11]) Let $\left\{s_{n}\right\}$ be a sequence of nonnegative real numbers satisfying

$$
s_{n+1}=\left(1-\alpha_{n}\right) s_{n}+\alpha_{n} \beta_{n}, \quad \forall n \geq 0,
$$

where $\left\{\alpha_{n}\right\},\left\{\beta_{n}\right\}$ satisfy the conditions

(1) $\left\{\alpha_{n}\right\} \subset[0,1], \sum_{n=1}^{\infty} \alpha_{n}=\infty$,

(2) $\lim \sup _{n \rightarrow \infty} \beta_{n} \leq 0$ or $\sum_{n=1}^{\infty}\left|\alpha_{n} \beta_{n}\right|<\infty$.

Then $\lim _{n \rightarrow \infty} s_{n}=0$. 
Lemma 2.5 (See [12]) Let E be a uniformly convex Banach space, let $C$ be a nonempty closed convex subset of $E$ and let $S: C \rightarrow C$ be a nonexpansive mapping. Then $I-S$ is demi-closed at zero.

In 2009, Kangtunykarn and Suantai [13] defined an $S$-mapping and proved their lemmas as follows.

Definition 2.1 Let $C$ be a nonempty convex subset of a real Banach space. Let $\left\{T_{i}\right\}_{i=1}^{N}$ be a finite family of nonexpanxive mappings of $C$ into itself. For each $j=1,2, \ldots, N$, let $\alpha_{j}=$ $\left(\alpha_{1}^{j}, \alpha_{2}^{j}, \alpha_{3}^{j}\right) \in I \times I \times I$, where $I \in[0,1]$ and $\alpha_{1}^{j}+\alpha_{2}^{j}+\alpha_{3}^{j}=1$. Define the mapping $S: C \rightarrow C$ as follows:

$$
\begin{aligned}
& U_{0}=I, \\
& U_{1}=\alpha_{1}^{1} T_{1} U_{0}+\alpha_{2}^{1} U_{0}+\alpha_{3}^{1} I, \\
& U_{2}=\alpha_{1}^{2} T_{2} U_{1}+\alpha_{2}^{2} U_{1}+\alpha_{3}^{2} I, \\
& U_{3}=\alpha_{1}^{3} T_{3} U_{2}+\alpha_{2}^{3} U_{2}+\alpha_{3}^{3} I, \\
& \vdots \\
& U_{N-1}=\alpha_{1}^{N-1} T_{N-1} U_{N-2}+\alpha_{2}^{N-1} U_{N-2}+\alpha_{3}^{N-1} I, \\
& S=U_{N}=\alpha_{1}^{N} T_{N} U_{N-1}+\alpha_{2}^{N} U_{N-1}+\alpha_{3}^{N} I .
\end{aligned}
$$

This mapping is called an $S$-mapping generated by $T_{1}, T_{2}, \ldots, T_{N}$ and $\alpha_{1}, \alpha_{2}, \ldots, \alpha_{N}$.

Lemma 2.6 Let $C$ be a nonempty closed convex subset of strictly convex Banach space. Let $\left\{T_{i}\right\}_{i=1}^{N}$ be a finite family of nonexpanxive mappings of $C$ into itself with $\bigcap_{i=1}^{N} F\left(T_{i}\right) \neq \emptyset$ and let $\alpha_{j}=\left(\alpha_{1}^{j}, \alpha_{2}^{j}, \alpha_{3}^{j}\right) \in I \times I \times I, j=1,2,3, \ldots, N$, where $I=[0,1], \alpha_{1}^{j}+\alpha_{2}^{j}+\alpha_{3}^{j}=1, \alpha_{1}^{j} \in(0,1)$ for all $j=1,2, \ldots, N-1, \alpha_{1}^{N} \in(0,1], \alpha_{2}^{j}, \alpha_{3}^{j} \in[0,1)$ for all $j=1,2, \ldots, N$. Let $S$ be a mapping generated by $T_{1}, T_{2}, \ldots, T_{N}$ and $\alpha_{1}, \alpha_{2}, \ldots, \alpha_{N}$. Then $F(S)=\bigcap_{i=1}^{N} F\left(T_{i}\right)$.

Remark 2.7 It is easy to see that the mapping $S$ is a nonexpansive mapping.

Lemma 2.8 Let $C$ be a nonempty closed convex subset of $H$. Let $T: C \rightarrow C$ be a $\kappa$-strictly pseudononspreading mapping with $F(T) \neq \emptyset$. Then $F(T)=V I(C,(I-T))$.

Proof It is easy to see that $F(T) \subseteq V I(C,(I-T))$. Put $A=I-T$. Let $z \in V I(C, A)$ and $z^{*} \in$ $F(T)$. Since $z \in V I(C, A)$, we have

$$
\langle y-z,(I-T) z\rangle \geq 0, \quad \forall y \in C .
$$

Since $T$ is a $\kappa$-strictly pseudononspreading mapping, we have

$$
\begin{aligned}
\left\|T z-T z^{*}\right\|^{2} & =\left\|(I-A) z-(I-A) z^{*}\right\|^{2} \\
& =\left\|\left(z-z^{*}\right)-\left(A z-A z^{*}\right)\right\|^{2} \\
& =\left\|z-z^{*}\right\|^{2}+\left\|A z-A z^{*}\right\|^{2}-2\left\langle z-z^{*}, A z-A z^{*}\right\rangle
\end{aligned}
$$




$$
\begin{aligned}
& =\left\|z-z^{*}\right\|^{2}+\|A z\|^{2}-2\left\langle z-z^{*}, A z\right\rangle \\
& \leq\left\|z-z^{*}\right\|^{2}+\kappa\left\|(I-T) z-(I-T) z^{*}\right\|^{2}+2\left\langle z-T z, z^{*}-T z^{*}\right\rangle \\
& =\left\|z-z^{*}\right\|^{2}+\kappa\|(I-T) z\|^{2}
\end{aligned}
$$

which implies that

$$
\begin{aligned}
\frac{1-\kappa}{2}\|z-T z\|^{2} & \leq\left\langle z-z^{*},(I-T) z\right\rangle \\
& =-\left\langle z^{*}-z,(I-T) z\right\rangle \\
& \leq 0 .
\end{aligned}
$$

Then we have $z \in F(T)$. Therefore $V I(C,(I-T)) \subseteq F(T)$. Hence $V I(C,(I-T))=F(T)$.

Remark 2.9 From Lemmas 2.2 and 2.8, we have $F(T)=F\left(P_{C}(I-\lambda(I-T))\right), \forall \lambda>0$.

Example 2.1 Let $T:[-1,1] \rightarrow[-1,1]$ be defined by

$$
T x= \begin{cases}\frac{x+4}{5} & \text { if } x \in[0,1], \\ \frac{4-x}{5} & \text { if } x \in[-1,0) .\end{cases}
$$

To see that $T$ is $\kappa$-strictly pseudononspreading, if for all $x, y \in[0,1]$, then we have $T x=\frac{x+4}{5}$ and $T y=\frac{y+4}{5}$. From the definition of $T$, we have

$$
\begin{aligned}
|T x-T y|^{2} & =\left|\frac{x+4}{5}-\frac{y+4}{5}\right|^{2} \\
=\frac{1}{25}|x-y|^{2} & \\
|(I-T) x-(I-T) y|^{2} & =|x-T x-(y-T y)|^{2} \\
& =\left|x-\frac{x+4}{5}-\left(y-\frac{y+4}{5}\right)\right|^{2} \\
& =\left|\frac{4 x-4}{5}-\frac{4 y-4}{5}\right|^{2} \\
& =\left|\frac{4 x-4 y}{5}\right|^{2} \\
& =\frac{16}{25}|x-y|^{2} \geq 0
\end{aligned}
$$

and

$$
\begin{aligned}
2\langle x-T x, y-T y\rangle & =2\left\langle x-\frac{x+4}{5}, y-\frac{y+4}{5}\right\rangle=2\left\langle\frac{4 x-4}{5}, \frac{4 y-4}{5}\right\rangle \\
& =\frac{32}{25}(x-1)(y-1) \geq 0 . \quad(\text { Since } 0 \leq x, y \leq 1,(x-1)(y-1) \geq 0 .)
\end{aligned}
$$


From the above, then there exists $\kappa \in[0,1)$ such that

$$
\begin{aligned}
|x-y|^{2}+\kappa|(I-T) x-(I-T) y|^{2}+2\langle x-T x, y-T y\rangle & \geq|x-y|^{2} \\
& \geq \frac{1}{25}|x-y|^{2} \\
& =|T x-T y|^{2} .
\end{aligned}
$$

For every $x, y \in[-1,0)$, we have $T x=\frac{4-x}{5}, T y=\frac{4-y}{5}$. From the definition of $T$, we have

$$
\begin{aligned}
|T x-T y|^{2} & =\left|\frac{4-x}{5}-\frac{4-y}{5}\right|^{2} \\
& =\left|\frac{y-x}{5}\right|^{2} \\
& =\frac{1}{25}|x-y|^{2}, \\
|(I-T) x-(I-T) y|^{2} & =|x-T x-(y-T y)|^{2} \\
& =\left|x-\frac{4-x}{5}-\left(y-\frac{4-y}{5}\right)\right|^{2} \\
& =\left|\frac{6 x-4-(6 y-4)}{5}\right|^{2} \\
& =\frac{36}{25}|x-y|^{2} \geq 0
\end{aligned}
$$

and

$$
\begin{aligned}
2\langle x-T x, y-T y\rangle & =2\left\langle x-\frac{4-x}{5}, y-\frac{4-y}{5}\right\rangle \\
& =2\left\langle\frac{6 x-4}{5}, \frac{6 y-4}{5}\right\rangle \\
& =\frac{8}{25}(3 x-2)(3 y-2) \\
& =\frac{8}{25}(3 x(3 y-2)-2(3 y-2)) \\
& =\frac{8}{25}(9 x y-6 x-6 y+4) \\
& >0 . \quad(\text { Since }-1 \leq x, y<0,9 x y,-6 x,-6 y>0 .)
\end{aligned}
$$

From the above, then there exists $\kappa \in[0,1)$ such that

$$
\begin{aligned}
|x-y|^{2}+\kappa|(I-T) x-(I-T) y|^{2}+2\langle x-T x, y-T y\rangle & >|x-y|^{2} \\
& \geq \frac{1}{25}|x-y|^{2} \\
& =|T x-T y|^{2} .
\end{aligned}
$$


Finally, for every $x \in[0,1]$ and $y \in[-1,0)$, we have $T x=\frac{x+4}{5}$ and $T y=\frac{4-y}{5}$. From the definition of $T$, we have

$$
\begin{aligned}
&|T x-T y|^{2}=\left|\frac{x+4}{5}-\frac{4-y}{5}\right|^{2} \\
&=\frac{1}{25}|x+y|^{2}, \\
&|(I-T) x-(I-T) y|^{2}=|x-T x-(y-T y)|^{2} \\
&=\left|x-\frac{x+4}{5}-\left(y-\frac{4-y}{5}\right)\right|^{2} \\
&=\left|\frac{4 x-4-(6 y-4)}{5}\right|^{2} \\
&=\frac{1}{25}|4 x-6 y|^{2} \geq 0
\end{aligned}
$$

and

$$
\begin{aligned}
2\langle x-T x, y-T y\rangle & =2\left\langle x-\frac{x+4}{5}, y-\frac{4-y}{5}\right\rangle \\
& =2\left\langle\frac{4 x-4}{5}, \frac{6 y-4}{5}\right\rangle \\
& =\frac{16}{25}(x-1)(3 y-2) \\
& =\frac{16}{25}(3 y(x-1)-2(x-1)) \\
& =\frac{16}{25}(3 y(x-1)+2(1-x)) \geq 0 .
\end{aligned}
$$

(Since $0 \leq x \leq 1$ and $-1 \leq y<0$, then $3 y(x-1), 2(1-x) \geq 0$.)

From the above, then there exists $\kappa \in[0,1)$ such that

$$
\begin{aligned}
|x-y|^{2}+\kappa|(I-T) x-(I-T) y|^{2}+2\langle x-T x, y-T y\rangle & \geq|x-y|^{2} \\
& =x^{2}-2 x y+y^{2} \\
& =x^{2}+2 x y+y^{2}-4 x y \\
& \geq(x+y)^{2} \quad(\text { Since }-4 x y \geq 0 .) \\
& \geq \frac{1}{25}|x+y|^{2} \\
& =|T x-T y|^{2} .
\end{aligned}
$$

Then, for all $x, y \in[-1,1]$, we have

$$
|T x-T y|^{2} \leq|x-y|^{2}+\kappa|(I-T) x-(I-T) y|^{2}+2\langle x-T x, y-T y\rangle
$$

for some $\kappa \in[0,1)$. Hence $T$ is a $\kappa$-strictly pseudononspreading mapping. Observe that $1 \in F(T)$. From Lemma 2.8, we have $1 \in V I([-1,1], I-T)$. 


\section{Main results}

Theorem 3.1 Let $H$ be a real Hilbert space and let $C$ be a nonempty closed convex subset of $H$. For every $i=1,2, \ldots, N$, let $B_{i}: C \rightarrow H$ be $\delta_{i}$-inverse strongly monotone mappings and let $T: C \rightarrow C$ be a $\kappa$-strictly pseudononspreading mapping for some $\kappa \in[0,1)$. Let $G_{i}: C \rightarrow C$ be defined by $G_{i} x=P_{C}\left(I-\eta B_{i}\right) x$ for every $x \in C$ and $\eta \in\left(0,2 \delta_{i}\right)$ for every $i=$ $1,2, \ldots, N$, and let $\delta_{j}=\left(\alpha_{1}^{j}, \alpha_{2}^{j}, \alpha_{3}^{j}\right) \in I \times I \times I, j=1,2,3, \ldots, N$, where $I=[0,1], \alpha_{1}^{j}+\alpha_{2}^{j}+\alpha_{3}^{j}=$ $1, \alpha_{1}^{j} \in(0,1)$ for all $j=1,2, \ldots, N-1, \alpha_{1}^{N} \in(0,1], \alpha_{2}^{j}, \alpha_{3}^{j} \in[0,1)$ for all $j=1,2, \ldots, N$. Let $S: C \rightarrow C$ be the $S$-mapping generated by $G_{1}, G_{2}, \ldots, G_{N}$ and $\delta_{1}, \delta_{2}, \ldots, \delta_{N}$. Assume that $\mathfrak{F}=F(T) \cap \bigcap_{i=1}^{N} V I\left(C, B_{i}\right) \neq \emptyset$. For every $n \in \mathbb{N}, i=1,2, \ldots, N$, let $x_{1}, u \in C$ and $\left\{x_{n}\right\}$ be a sequence generated by

$$
x_{n+1}=\alpha_{n} u+\beta_{n} P_{C}\left(I-\lambda_{n}(I-T)\right) x_{n}+\gamma_{n} S x_{n}, \quad \forall n \in \mathbb{N},
$$

where $\left\{\alpha_{n}\right\},\left\{\beta_{n}\right\},\left\{\gamma_{n}\right\},\left\{\lambda_{n}\right\} \subset(0,1)$ such that $\alpha_{n}+\beta_{n}+\gamma_{n}=1, \beta_{n} \in[c, d] \subset(0,1),\left\{\lambda_{n}\right\} \subset$ $(0,1-\kappa)$ and suppose the following conditions hold:

(i) $\lim _{n \rightarrow \infty} \alpha_{n}=0$ and $\sum_{n=0}^{\infty} \alpha_{n}=\infty$,

(ii) $\sum_{n=1}^{\infty} \lambda_{n}<\infty$,

(iii) $\sum_{n=1}^{\infty}\left|\lambda_{n+1}-\lambda_{n}\right|, \sum_{n=1}^{\infty}\left|\gamma_{n+1}-\gamma_{n}\right|, \sum_{n=1}^{\infty}\left|\alpha_{n+1}-\alpha_{n}\right|, \sum_{n=1}^{\infty}\left|\beta_{n+1}-\beta_{n}\right|<\infty$.

Then the sequence $\left\{x_{n}\right\}$ converges strongly to $z=P_{\mathfrak{F}} u$.

Proof Let $x^{*} \in \mathfrak{F}$. First, we show that $\left\|P_{C}\left(I-\lambda_{n} A\right) x_{n}-x^{*}\right\| \leq\left\|x_{n}-x^{*}\right\|$, where $A=I-T$. From Remark 2.9, we have $x^{*} \in F\left(P_{C}\left(I-\lambda_{n} A\right)\right)$. From the nonexpansiveness of $P_{C}$, we have

$$
\begin{aligned}
\left\|P_{C}\left(I-\lambda_{n} A\right) x_{n}-x^{*}\right\|^{2} & =\left\|P_{C}\left(I-\lambda_{n} A\right) x_{n}-P_{C}\left(I-\lambda_{n} A\right) x^{*}\right\|^{2} \\
& \leq\left\|\left(I-\lambda_{n} A\right) x_{n}-\left(I-\lambda_{n} A\right) x^{*}\right\|^{2} .
\end{aligned}
$$

Since $T$ is a $\kappa$-strictly pseudononspreading mapping and $A=I-T$, we have

$$
\begin{aligned}
\left\|T x_{n}-T x^{*}\right\|^{2} & =\left\|(I-A) x_{n}-(I-A) x^{*}\right\|^{2} \\
& =\left\|\left(x_{n}-x^{*}\right)-\left(A x_{n}-A x^{*}\right)\right\|^{2} \\
& =\left\|x_{n}-x^{*}\right\|^{2}-2\left\langle x_{n}-x^{*}, A x_{n}\right\rangle+\left\|A x_{n}\right\|^{2} \\
& \leq\left\|x_{n}-x^{*}\right\|^{2}+\kappa\left\|A x_{n}-A x^{*}\right\|^{2}+2\left\langle A x_{n}, A x^{*}\right\rangle \\
& =\left\|x_{n}-x^{*}\right\|^{2}+\kappa\left\|A x_{n}\right\|^{2},
\end{aligned}
$$

which implies that

$$
(1-\kappa)\left\|A x_{n}\right\|^{2} \leq 2\left\langle x_{n}-x^{*}, A x_{n}\right\rangle .
$$

From (3.3), we have

$$
\begin{aligned}
\left\|\left(I-\lambda_{n} A\right) x_{n}-\left(I-\lambda_{n} A\right) x^{*}\right\|^{2} & =\left\|\left(x_{n}-x^{*}\right)-\lambda_{n}\left(A x_{n}-A x^{*}\right)\right\|^{2} \\
& =\left\|x_{n}-x^{*}\right\|^{2}-2 \lambda_{n}\left\langle x_{n}-x^{*}, A x_{n}\right\rangle+\lambda_{n}^{2}\left\|A x_{n}\right\|^{2} \\
& \leq\left\|x_{n}-x^{*}\right\|^{2}-\lambda_{n}(1-\kappa)\left\|A x_{n}\right\|^{2}+\lambda_{n}^{2}\left\|A x_{n}\right\|^{2}
\end{aligned}
$$




$$
\begin{aligned}
& =\left\|x_{n}-x^{*}\right\|^{2}-\lambda_{n}\left((1-\kappa)-\lambda_{n}\right)\left\|A x_{n}\right\|^{2} \\
& \leq\left\|x_{n}-x^{*}\right\|^{2} .
\end{aligned}
$$

From (3.4) and (3.2), we can imply that

$$
\left\|P_{C}\left(I-\lambda_{n} A\right) x_{n}-x^{*}\right\| \leq\left\|x_{n}-x^{*}\right\| .
$$

Next, we will show that the mapping $G_{i}$ is a nonexpansive mapping for every $i=1,2, \ldots, N$. Let $x, y \in H$. Since $B_{i}$ is $\delta_{i}$-inverse strongly monotone and $0<\eta<2 \delta_{i}$, for every $i=$ $1,2, \ldots, N$, we have

$$
\begin{aligned}
\left\|\left(I-\eta B_{i}\right) x-\left(I-\eta B_{i}\right) y\right\|^{2} & =\left\|x-y-\eta\left(B_{i} x-B_{i} y\right)\right\|^{2} \\
& =\|x-y\|^{2}-2 \eta\left\langle x-y, B_{i} x-B_{i} y\right\rangle+\eta^{2}\left\|B_{i} x-B_{i} y\right\|^{2} \\
& \leq\|x-y\|^{2}-2 \delta_{i} \eta\left\|B_{i} x-B_{i} y\right\|^{2}+\eta^{2}\left\|B_{i} x-B_{i} y\right\|^{2} \\
& =\|x-y\|^{2}+\eta\left(\eta-2 \delta_{i}\right)\left\|B_{i} x-B_{i} y\right\|^{2} \\
& \leq\|x-y\|^{2} .
\end{aligned}
$$

Thus $\left(I-\eta B_{i}\right)$ is a nonexpansive mapping for every $i=1,2, \ldots, N$. The proof of the above result can be also found in Imnang and Suantai [14]. From the definition of $G_{i}$, we have $G_{i}=$ $P_{C}\left(I-\eta B_{i}\right)$ are nonexpansive mappings for all $i=1,2, \ldots, N$. Since $x^{*} \in \mathfrak{F}$, by Lemma 2.2, we have

$$
x^{*}=G_{i} x^{*}=P_{C}\left(I-\eta B_{i}\right) x^{*}, \quad \forall i=1,2, \ldots, N .
$$

From Lemma 2.6, we have $x^{*} \in F(S)$. Next, we will show that $\left\{x_{n}\right\}$ is bounded. From the definition of $x_{n}$ and (3.5), we have

$$
\begin{aligned}
\left\|x_{n+1}-x^{*}\right\| & =\left\|\alpha_{n}\left(u-x^{*}\right)+\beta_{n}\left(P_{C}\left(I-\lambda_{n}(I-T)\right) x_{n}-x^{*}\right)+\gamma_{n}\left(S x_{n}-x^{*}\right)\right\| \\
& \leq \alpha_{n}\left\|u-x^{*}\right\|+\beta_{n}\left\|P_{C}\left(I-\lambda_{n}(I-T)\right) x_{n}-x^{*}\right\|+\gamma_{n}\left\|S x_{n}-x^{*}\right\| \\
& \leq \alpha_{n}\left\|u-x^{*}\right\|+\left(1-\alpha_{n}\right)\left\|x_{n}-x^{*}\right\| .
\end{aligned}
$$

Put $K=\max \left\{\left\|u-x^{*}\right\|,\left\|x_{1}-x^{*}\right\|\right\}$. From (3.8) we can show by induction that $\left\|x_{n}-x^{*}\right\| \leq K$, $\forall n \in \mathbb{N}$. This implies that $\left\{x_{n}\right\}$ is bounded and so are $\left\{S x_{n}\right\},\left\{P_{C}\left(I-\lambda_{n}(I-T)\right) x_{n}\right\}$. Next, we will show that

$$
\left\|T x_{n}-x^{*}\right\| \leq \frac{1+\kappa}{1-\kappa}\left\|x_{n}-x^{*}\right\| .
$$

Since $T$ is $\kappa$-strictly pseudononspreading, we have

$$
\begin{aligned}
\left\|T x_{n}-x^{*}\right\|^{2} & \leq\left\|x_{n}-x^{*}\right\|^{2}+\kappa\left\|(I-T) x_{n}-(I-T) x^{*}\right\|^{2}+2\left\langle x_{n}-T x_{n}, x^{*}-T x^{*}\right\rangle \\
& =\left\|x_{n}-x^{*}\right\|^{2}+\kappa\left\|\left(x_{n}-x^{*}\right)-\left(T x_{n}-x^{*}\right)\right\|^{2} \\
& =\left\|x_{n}-x^{*}\right\|^{2}+\kappa\left(\left\|x_{n}-x^{*}\right\|^{2}+\left\|T x_{n}-x^{*}\right\|^{2}-2\left(x_{n}-x^{*}, T x_{n}-x^{*}\right\rangle\right),
\end{aligned}
$$


which implies that

$$
(1-\kappa)\left\|T x_{n}-x^{*}\right\|^{2} \leq(1+\kappa)\left\|x_{n}-x^{*}\right\|^{2}+2 \kappa\left\|x_{n}-x^{*}\right\|\left\|T x_{n}-x^{*}\right\| .
$$

Putting $A=\left\|T x_{n}-x^{*}\right\|$ and $B=\left\|x_{n}-x^{*}\right\|$ in (3.10), we have

$$
\begin{aligned}
0 & \geq(1-\kappa) A^{2}-(1+\kappa) B^{2}-2 \kappa A B \\
& =(1-\kappa) A^{2}-\kappa A B-\left((1+\kappa) B^{2}+\kappa A B\right) \\
& =(1-\kappa) A^{2}-\kappa A B+A B-\left((1+\kappa) B^{2}+\kappa A B+A B\right) \\
& =(1-\kappa)\left(A^{2}+A B\right)-\left((1+\kappa)\left(B^{2}+A B\right)\right) \\
& =(1-\kappa) A(A+B)-((1+\kappa) B(B+A)),
\end{aligned}
$$

which implies that

$$
A \leq \frac{1+\kappa}{1-\kappa} B
$$

From (3.11) we have (3.9). Since $\left\|x_{n}-x^{*}\right\| \leq K, \forall n \in \mathbb{N}$ and (3.9), we have $\left\{T x_{n}\right\}$ is bounded.

Next, we will show that

$$
\lim _{n \rightarrow \infty}\left\|x_{n+1}-x_{n}\right\|=0
$$

From the definition of $x_{n}$, we have

$$
\begin{aligned}
\left\|x_{n+1}-x_{n}\right\| \leq & \| \alpha_{n} u+\beta_{n} P_{C}\left(I-\lambda_{n}(I-T)\right) x_{n}+\gamma_{n} S x_{n} \\
& -\alpha_{n-1} u-\beta_{n-1} P_{C}\left(I-\lambda_{n-1}(I-T)\right) x_{n-1}-\gamma_{n-1} S x_{n-1} \| \\
= & \| \alpha_{n} u+\beta_{n} P_{C}\left(I-\lambda_{n}(I-T)\right) x_{n}-\beta_{n} P_{C}\left(I-\lambda_{n-1}(I-T)\right) x_{n-1} \\
& +\beta_{n} P_{C}\left(I-\lambda_{n-1}(I-T)\right) x_{n-1}+\gamma_{n} S x_{n}-\gamma_{n} S x_{n-1}+\gamma_{n} S x_{n-1} \\
& -\alpha_{n-1} u-\beta_{n-1} P_{C}\left(I-\lambda_{n-1}(I-T)\right) x_{n-1}-\gamma_{n-1} S x_{n-1} \| \\
\leq & \left|\alpha_{n}-\alpha_{n-1}\right|\|u\|+\beta_{n}\left\|P_{C}\left(I-\lambda_{n}(I-T)\right) x_{n}-P_{C}\left(I-\lambda_{n-1}(I-T)\right) x_{n-1}\right\| \\
& +\left|\beta_{n}-\beta_{n-1}\right|\left\|P_{C}\left(I-\lambda_{n-1}(I-T)\right) x_{n-1}\right\|+\gamma_{n}\left\|S x_{n}-S x_{n-1}\right\| \\
& +\left|\gamma_{n}-\gamma_{n-1}\right|\left\|S x_{n-1}\right\| \\
\leq & \left|\alpha_{n}-\alpha_{n-1}\right|\|u\|+\beta_{n} \|\left(x_{n}-x_{n-1}\right)-\lambda_{n}(I-T) x_{n}+\lambda_{n}(I-T) x_{n-1} \\
& -\lambda_{n}(I-T) x_{n-1}+\lambda_{n-1}(I-T) x_{n-1} \| \\
& +\left|\beta_{n}-\beta_{n-1}\right|\left\|P_{C}\left(I-\lambda_{n-1}(I-T)\right) x_{n-1}\right\| \\
& +\gamma_{n}\left\|x_{n}-x_{n-1}\right\|+\left|\gamma_{n}-\gamma_{n-1}\right|\left\|S x_{n-1}\right\| \\
\leq & \left|\alpha_{n}-\alpha_{n-1}\right|\|u\|+\beta_{n}\left(\left\|x_{n}-x_{n-1}\right\|+\lambda_{n}\left\|(I-T) x_{n}-(I-T) x_{n-1}\right\|\right. \\
& \left.+\left|\lambda_{n}-\lambda_{n-1}\right|\left\|(I-T) x_{n-1}\right\|\right)+\left|\beta_{n}-\beta_{n-1}\right|\left\|P C\left(I-\lambda_{n-1}(I-T)\right) x_{n-1}\right\| \\
& +\gamma_{n}\left\|x_{n}-x_{n-1}\right\|+\left|\gamma_{n}-\gamma_{n-1}\right|\left\|S x_{n-1}\right\|
\end{aligned}
$$




$$
\begin{aligned}
\leq & \left|\alpha_{n}-\alpha_{n-1}\right| L+\left(1-\alpha_{n}\right) \| x_{n}-x_{n-1}||+\lambda_{n} L \\
& +\left|\lambda_{n}-\lambda_{n-1}\right| L+\left|\beta_{n}-\beta_{n-1}\right| L+\left|\gamma_{n}-\gamma_{n-1}\right| L,
\end{aligned}
$$

where $L=\max _{n \in \mathbb{N}}\left\{\|u\|,\left\|(I-T) x_{n}-(I-T) x_{n-1}\right\|,\left\|(I-T) x_{n}\right\|,\left\|P_{C}\left(I-\lambda_{n}(I-T)\right) x_{n}\right\|,\left\|S x_{n}\right\|\right\}$. From Lemma 2.3 and conditions (i)-(iii), we have (3.12). Next, we will show that

$$
\lim _{n \rightarrow \infty}\left\|x_{n}-P_{C}\left(I-\lambda_{n}(I-T)\right) x_{n}\right\|=0 .
$$

From the definition of $x_{n}$ and (3.5), we have

$$
\begin{aligned}
\left\|x_{n+1}-x^{*}\right\|^{2}= & \left\|\alpha_{n}\left(u-x^{*}\right)+\beta_{n}\left(P_{C}\left(I-\lambda_{n}(I-T)\right) x_{n}-x^{*}\right)+\gamma_{n}\left(S x_{n}-x^{*}\right)\right\|^{2} \\
\leq & \alpha_{n}\left\|u-x^{*}\right\|^{2}+\beta_{n}\left\|P_{C}\left(I-\lambda_{n}(I-T)\right) x_{n}-x^{*}\right\|^{2}+\gamma_{n}\left\|S x_{n}-x^{*}\right\|^{2} \\
& -\beta_{n} \gamma_{n}\left\|P_{C}\left(I-\lambda_{n}(I-T)\right) x_{n}-S x_{n}\right\|^{2} \\
\leq & \alpha_{n}\left\|u-x^{*}\right\|^{2}+\left(1-\alpha_{n}\right)\left\|x_{n}-x^{*}\right\|^{2} \\
& -\beta_{n} \gamma_{n}\left\|P_{C}\left(I-\lambda_{n}(I-T)\right) x_{n}-S x_{n}\right\|^{2} \\
\leq & \alpha_{n}\left\|u-x^{*}\right\|^{2}+\left\|x_{n}-x^{*}\right\|^{2} \\
& -\beta_{n} \gamma_{n}\left\|P_{C}\left(I-\lambda_{n}(I-T)\right) x_{n}-S x_{n}\right\|^{2},
\end{aligned}
$$

which implies that

$$
\begin{aligned}
\beta_{n} \gamma_{n}\left\|P_{C}\left(I-\lambda_{n}(I-T)\right) x_{n}-S x_{n}\right\|^{2} \leq & \alpha_{n}\left\|u-x^{*}\right\|^{2}+\left\|x_{n}-x^{*}\right\|^{2}-\left\|x_{n+1}-x^{*}\right\|^{2} \\
\leq & \alpha_{n}\left\|u-x^{*}\right\|^{2}+\left(\left\|x_{n}-x^{*}\right\|\right. \\
& \left.+\left\|x_{n+1}-x^{*}\right\|\right)\left\|x_{n+1}-x_{n}\right\| .
\end{aligned}
$$

From (3.15) and (3.12), we have

$$
\lim _{n \rightarrow \infty}\left\|P_{C}\left(I-\lambda_{n}(I-T)\right) x_{n}-S x_{n}\right\|=0 .
$$

Since

$$
x_{n+1}-P_{C}\left(I-\lambda_{n}(I-T)\right) x_{n}=\alpha_{n}\left(u-P_{C}\left(I-\lambda_{n}(I-T)\right) x_{n}\right)+\gamma_{n}\left(S x_{n}-P_{C}\left(I-\lambda_{n}(I-T)\right) x_{n}\right),
$$

from condition (i) and (3.16), we have

$$
\lim _{n \rightarrow \infty}\left\|x_{n+1}-P_{C}\left(I-\lambda_{n}(I-T)\right) x_{n}\right\|=0 .
$$

Since

$$
\left\|x_{n}-P_{C}\left(I-\lambda_{n}(I-T)\right) x_{n}\right\| \leq\left\|x_{n}-x_{n+1}\right\|+\left\|x_{n+1}-P_{C}\left(I-\lambda_{n}(I-T)\right) x_{n}\right\|,
$$

from (3.12) and (3.17), we have (3.14). Since

$$
x_{n+1}-x_{n}=\alpha_{n}\left(u-x_{n}\right)+\beta_{n}\left(P_{C}\left(I-\lambda_{n}(I-T)\right) x_{n}-x_{n}\right)+\gamma_{n}\left(S x_{n}-x_{n}\right),
$$


from condition (i) (3.12) and (3.14), we have

$$
\lim _{n \rightarrow \infty}\left\|S x_{n}-x_{n}\right\|=0
$$

Next, we will show that

$$
\limsup _{n \rightarrow \infty}\left\langle u-z_{0}, x_{n}-z_{0}\right\rangle \leq 0
$$

where $z_{0}=P_{\mathfrak{F}} u$. To show this equality, take a subsequence $\left\{x_{n_{m}}\right\}$ of $\left\{x_{n}\right\}$ such that

$$
\limsup _{n \rightarrow \infty}\left\langle u-z_{0}, x_{n}-z_{0}\right\rangle=\lim _{m \rightarrow \infty}\left\langle u-z_{0}, x_{n_{m}}-z_{0}\right\rangle
$$

Without loss of generality, we may assume that $x_{n_{m}} \rightarrow \omega$ as $m \rightarrow \infty$ where $\omega \in C$. We shall show that $\omega \in F(T)$. From Remark 2.9, we have $F(T)=F\left(P_{C}\left(I-\lambda_{n_{m}}(I-T)\right)\right)$. Assume that $\omega \neq P_{C}\left(I-\lambda_{n_{m}}(I-T)\right) \omega$. Since $x_{n_{m}} \rightarrow \omega$ as $m \rightarrow \infty$, by Opial's property, (3.14) and condition (ii), we have

$$
\begin{aligned}
\liminf _{m \rightarrow \infty}\left\|x_{n_{m}}-\omega\right\|< & \liminf _{m \rightarrow \infty}\left\|x_{n_{m}}-P_{C}\left(I-\lambda_{n_{m}}(I-T)\right) \omega\right\| \\
\leq & \liminf _{m \rightarrow \infty}\left(\left\|x_{n_{m}}-P_{C}\left(I-\lambda_{n_{m}}(I-T)\right) x_{n_{m}}\right\|\right. \\
& \left.\quad+\left\|P_{C}\left(I-\lambda_{n_{m}}(I-T)\right) x_{n_{m}}-P_{C}\left(I-\lambda_{n_{m}}(I-T)\right) \omega\right\|\right) \\
\leq & \liminf _{m \rightarrow \infty}\left(\left\|x_{n_{m}}-P_{C}\left(I-\lambda_{n_{m}}(I-T)\right) x_{n_{m}}\right\|\right. \\
& \left.\quad+\left\|x_{n_{m}}-\omega\right\|+\lambda_{n_{m}}\left\|(I-T) x_{n_{m}}-(I-T) \omega\right\|\right) \\
\leq & \liminf _{m \rightarrow \infty}\left\|x_{n_{m}}-\omega\right\| .
\end{aligned}
$$

This is a contradiction. Then $\omega \in F(T)$. From (3.18), we have

$$
\lim _{m \rightarrow \infty}\left\|S x_{n_{m}}-x_{n_{m}}\right\|=0
$$

From the nonexpansiveness of $S, x_{n_{m}} \rightarrow \omega$ as $m \rightarrow \infty$ and Lemma 2.5, we can imply that

$$
\omega \in F(S) \text {. }
$$

Since $P_{C}\left(I-\eta B_{i}\right) x=G_{i} x$ for every $x \in C$ and $i=1,2, \ldots, N$, by Lemma 2.2 , we have

$$
V I\left(C, B_{i}\right)=F\left(P_{C}\left(I-\eta B_{i}\right)\right)=F\left(G_{i}\right), \quad \forall i=1,2, \ldots, N
$$

By Lemma 2.6, we have

$$
F(S)=\bigcap_{i=1}^{N} F\left(G_{i}\right)=\bigcap_{i=1}^{N} V I\left(C, B_{i}\right) .
$$

From (3.21) and (3.22), we have $\omega \in \bigcap_{i=1}^{N} V I\left(C, B_{i}\right)$. Hence $\omega \in \mathfrak{F}$. Since $x_{n_{m}} \rightarrow \omega$ as $m \rightarrow \infty$ and $\omega \in \mathfrak{F}$, we have

$$
\limsup _{n \rightarrow \infty}\left\langle u-z_{0}, x_{n}-z_{0}\right\rangle=\lim _{m \rightarrow \infty}\left\langle u-z_{0}, x_{n_{m}}-z_{0}\right\rangle=\left\langle u-z_{0}, \omega-z_{0}\right\rangle \leq 0
$$


Finally, we show that $\left\{x_{n}\right\}$ converges strongly to $z_{0}=P_{\mathfrak{F}} u$. From the definition of $x_{n}$ and (3.5), we have

$$
\begin{aligned}
\left\|x_{n+1}-z_{0}\right\|^{2}= & \left\|\alpha_{n}\left(u-z_{0}\right)+\beta_{n}\left(P_{C}\left(I-\lambda_{n}(I-T)\right) x_{n}-z_{0}\right)+\gamma_{n}\left(S x_{n}-z_{0}\right)\right\|^{2} \\
\leq & \left\|\beta_{n}\left(P_{C}\left(I-\lambda_{n}(I-T)\right) x_{n}-z_{0}\right)+\gamma_{n}\left(S x_{n}-z_{0}\right)\right\|^{2} \\
& +2\left\langle\alpha_{n}\left(u-z_{0}\right), x_{n+1}-z_{0}\right\rangle \\
\leq & \beta_{n}\left\|P_{C}\left(I-\lambda_{n}(I-T)\right) x_{n}-z_{0}\right\|^{2}+\gamma_{n}\left\|S x_{n}-z_{0}\right\|^{2} \\
& +2 \alpha_{n}\left\langle u-z_{0}, x_{n+1}-z_{0}\right\rangle \\
\leq & \left(1-\alpha_{n}\right)\left\|x_{n}-z_{0}\right\|^{2}+2 \alpha_{n}\left\langle u-z_{0}, x_{n+1}-z_{0}\right\rangle .
\end{aligned}
$$

From (3.19) and Lemma 2.4, we have $\left\{x_{n}\right\}$ converges strongly to $z_{0}=P_{\mathfrak{F}} u$. This completes the proof.

The following result can be obtained from Theorem 3.1. We, therefore, omit the proof.

Corollary 3.2 Let $C$ be a nonempty closed convex subset of a real Hilbert space $H$. Let $B: C \rightarrow H$ be a $\delta$-inverse strongly monotone mapping and let $T: C \rightarrow C$ be a $\kappa$-strictly pseudononspreading mapping for some $\kappa \in[0,1)$. Assume that $\mathfrak{F}=F(T) \cap V I(C, B) \neq \emptyset$. For every $n \in \mathbb{N}$, let $x_{1}, u \in C$ and $\left\{x_{n}\right\}$ be a sequence generated by

$$
x_{n+1}=\alpha_{n} u+\beta_{n} P_{C}\left(I-\lambda_{n}(I-T)\right) x_{n}+\gamma_{n} P_{C}(I-\eta B) x_{n}, \quad \forall n \in \mathbb{N},
$$

where $\left\{\alpha_{n}\right\},\left\{\beta_{n}\right\},\left\{\gamma_{n}\right\},\left\{\lambda_{n}\right\} \subset(0,1)$ such that $\alpha_{n}+\beta_{n}+\gamma_{n}=1, \beta_{n} \in[c, d] \subset(0,1), \lambda_{n} \subset(0,1-$ $\kappa)$, and $\eta \in(0,2 \delta)$, and suppose that the following conditions hold:

(i) $\lim _{n \rightarrow \infty} \alpha_{n}=0$ and $\sum_{n=0}^{\infty} \alpha_{n}=\infty$,

(ii) $\sum_{n=1}^{\infty} \lambda_{n}<\infty$,

(iii) $\sum_{n=1}^{\infty}\left|\lambda_{n+1}-\lambda_{n}\right|, \sum_{n=1}^{\infty}\left|\gamma_{n+1}-\gamma_{n}\right|, \sum_{n=1}^{\infty}\left|\alpha_{n+1}-\alpha_{n}\right|, \sum_{n=1}^{\infty}\left|\beta_{n+1}-\beta_{n}\right|<\infty$.

Then the sequence $\left\{x_{n}\right\}$ converges strongly to $z=P_{\mathfrak{F}} u$.

\section{Application}

In this section, by using our main result, we prove strong a convergence theorem involving a strictly pseudononspreading mapping and a finite family of strictly pseudocontractive mappings. Before proving the next theorem, we need the following definition.

Definition 4.1 The mapping $T: C \rightarrow C$ is said to be strictly pseudocontractive [2] with the coefficient $\kappa \in[0,1)$ if

$$
\|T x-T y\|^{2} \leq\|x-y\|^{2}+\kappa\|(I-T) x-(I-T) y\|^{2} \quad \forall x, y \in C
$$

Remark 4.1 If $C$ is a nonempty closed convex subset of $H$ and $T: C \rightarrow C$ is a $\kappa$-strictly pseudocontractive mapping with $F(T) \neq \emptyset$, then $F(T)=V I(C,(I-T))$. To show this, put $A=I-T$. Let $z \in V I(C,(I-T))$ and $z^{*} \in F(T)$. Since $z \in V I(C,(I-T)),\langle y-z,(I-T) z\rangle \geq 0$, 
$\forall y \in C$. Since $T: C \rightarrow C$ is a $\kappa$-strictly pseudocontractive mapping, we have

$$
\begin{aligned}
\left\|T z-T z^{*}\right\|^{2} & =\left\|(I-A) z-(I-A) z^{*}\right\|^{2}=\left\|z-z^{*}-\left(A z-A z^{*}\right)\right\|^{2} \\
& =\left\|z-z^{*}\right\|^{2}-2\left\langle z-z^{*}, A z-A z^{*}\right\rangle+\left\|A z-A z^{*}\right\|^{2} \\
& =\left\|z-z^{*}\right\|^{2}-2\left\langle z-z^{*},(I-T) z\right\rangle+\|(I-T) z\|^{2} \\
& \leq\left\|z-z^{*}\right\|^{2}+\kappa\|(I-T) z\|^{2} .
\end{aligned}
$$

It implies that

$$
(1-\kappa)\|(I-T) z\|^{2} \leq 2\left|z-z^{*},(I-T) z\right\rangle \leq 0 .
$$

Then we have $z=T z$, therefore $z \in F(T)$. Hence $V I(C,(I-T)) \subseteq F(T)$. It is easy to see that $F(T) \subseteq V I(C,(I-T))$.

Remark 4.2 $A=I-T$ is a $\frac{1-\kappa}{2}$-inverse strongly monotone mapping. To show this, let $x, y \in C$, we have

$$
\begin{aligned}
\|T x-T y\|^{2} & =\|(I-A) x-(I-A) y\|^{2}=\|x-y-(A x-A y)\|^{2} \\
& =\|x-y\|^{2}-2\langle x-y, A x-A y\rangle+\|A x-A y\|^{2} \\
& \leq\|x-y\|^{2}+\kappa\|(I-T) x-(I-T) y\|^{2} \\
& =\|x-y\|^{2}+\kappa\|A x-A y\|^{2} .
\end{aligned}
$$

Then we have

$$
\langle x-y, A x-A y\rangle \geq \frac{1-\kappa}{2}\|A x-A y\|^{2} .
$$

Theorem 4.3 Let $H$ be a real Hilbert space and let $C$ be a nonempty closed convex subset of $H$. Let $T_{i}^{k}: C \rightarrow C$ be $\kappa_{i}$-strictly pseudocontractive mappings for every $i=1,2, \ldots, N$, and let $T: C \rightarrow C$ be a $\kappa$-strictly pseudononspreading mapping for some $\kappa \in[0,1)$. Let $F_{i}: C \rightarrow C$ be defined by $P_{C}\left(I-\eta\left(I-T_{i}^{k}\right)\right) x=F_{i} x$ for every $x \in C$ and $\eta \in\left(0,1-\kappa_{i}\right)$ for every $i=1,2, \ldots, N$, and let $\delta_{j}=\left(\alpha_{1}^{j}, \alpha_{2}^{j}, \alpha_{3}^{j}\right) \in I \times I \times I, j=1,2,3, \ldots, N$, where $I=[0,1]$, $\alpha_{1}^{j}+\alpha_{2}^{j}+\alpha_{3}^{j}=1, \alpha_{1}^{j} \in(0,1)$ for all $j=1,2, \ldots, N-1, \alpha_{1}^{N} \in(0,1], \alpha_{2}^{j}, \alpha_{3}^{j} \in[0,1)$ for all $j=$ $1,2, \ldots, N$. Let $S: C \rightarrow C$ be the $S$-mapping generated by $F_{1}, F_{2}, \ldots, F_{N}$ and $\delta_{1}, \delta_{2}, \ldots, \delta_{N}$. Assume that $\mathfrak{F}=F(T) \cap \bigcap_{i=1}^{N} F\left(T_{i}^{k}\right) \neq \emptyset$. For every $n \in \mathbb{N}, i=1,2, \ldots, N$, let $x_{1}, u \in C$ and $\left\{x_{n}\right\}$ be a sequence generated by

$$
x_{n+1}=\alpha_{n} u+\beta_{n} P_{C}\left(I-\lambda_{n}(I-T)\right) x_{n}+\gamma_{n} S x_{n}, \quad \forall n \in \mathbb{N},
$$

where $\left\{\alpha_{n}\right\},\left\{\beta_{n}\right\},\left\{\gamma_{n}\right\},\left\{\lambda_{n}\right\} \subset(0,1)$ such that $\alpha_{n}+\beta_{n}+\gamma_{n}=1, \beta_{n} \in[c, d] \subset(0,1), \lambda_{n} \subset(0,1-$ $\kappa)$ and suppose that the following conditions hold:

(i) $\lim _{n \rightarrow \infty} \alpha_{n}=0$ and $\sum_{n=0}^{\infty} \alpha_{n}=\infty$,

(ii) $\sum_{n=1}^{\infty} \lambda_{n}<\infty$,

(iii) $\sum_{n=1}^{\infty}\left|\lambda_{n+1}-\lambda_{n}\right|, \sum_{n=1}^{\infty}\left|\gamma_{n+1}-\gamma_{n}\right|, \sum_{n=1}^{\infty}\left|\alpha_{n+1}-\alpha_{n}\right|, \sum_{n=1}^{\infty}\left|\beta_{n+1}-\beta_{n}\right|<\infty$.

Then the sequence $\left\{x_{n}\right\}$ converges strongly to $z=P_{\mathfrak{F}} u$. 
Proof From Remark 4.2, we have $I-T_{i}^{k}$ is $\frac{1-\kappa_{i}}{2}$-inverse strongly monotone for every $i=$ $1,2, \ldots, N$. From Remark 4.1 and Lemma 2.2 , we have $F\left(T_{i}^{k}\right)=V I\left(C,\left(I-T_{i}^{k}\right)\right)=F\left(P_{C}(I-\right.$ $\left.\left.\eta\left(I-T_{i}^{k}\right)\right)\right)$ for every $i=1,2, \ldots, N$. Put $I-T_{i}^{k} \equiv B_{i}$ and $\delta_{i}=\frac{1-\kappa_{i}}{2}$ for every $i=1,2, \ldots, N$ in Theorem 3.1. The conclusion of Theorem 4.3 can be obtained from Theorem 3.1

\section{Competing interests}

The author declares that they have no competing interests.

\section{Acknowledgements}

This research was supported by the Research Administration Division of King Mongkut's Institute of Technology Ladkrabang.

Received: 2 September 2012 Accepted: 14 June 2013 Published: 2 July 2013

\section{References}

1. Kohsaka, F, Takahashi, W: Fixed point theorems for a class of nonlinear mappings related to maximal monotone operators in Banach spaces. Arch. Math. 91, 166-177 (2008)

2. Browder, FE, Petryshyn, WV: Construction of fixed points of nonlinear mappings in Hilbert spaces. J. Math. Anal. Appl. 20, 197-228 (1967)

3. Osilike, MO, Isiogugu, FO: Weak and strong convergence theorems for nonspreading-type mappings in Hilbert spaces. Nonlinear Anal. 74, 1814-1822 (2011)

4. Chang, SS, Joseph Lee, HW, Chan, CK: A new method for solving equilibrium problem fixed point problem and variational inequality problem with application to optimization. Nonlinear Anal. 70, 3307-3319 (2009)

5. Nadezhkina, N, Takahashi, W: Weak convergence theorem by an extragradient method for nonexpansive mappings and monotone mappings. J. Optim. Theory Appl. 128, 191-201 (2006)

6. Yao, JC, Chadli, O: Pseudomonotone complementarity problems and variational inequalities. In: Crouzeix, JP, Haddjissas, N, Schaible, S (eds.) Handbook of Generalized Convexity and Monotonicity, pp. 501-558. Springer, New York (2005)

7. Yao, Y, Yao, JC: On modified iterative method for nonexpansive mappings and monotone mappings. Appl. Math. Comput. 186(2), 1551-1558 (2007)

8. liduka, H, Takahashi, W: Weak convergence theorem by Cesàro means for nonexpansive mappings and inverse-strongly monotone mappings. J. Nonlinear Convex Anal. 7, 105-113 (2006)

9. Takahashi, W, Toyoda, M: Weak convergence theorems for nonexpansive mappings and monotone mappings. J. Optim. Theory Appl. 118, 417-428 (2003)

10. Takahashi, W: Nonlinear Functional Analysis. Yokohama Publishers, Yokohama (2000)

11. Xu, HK: An iterative approach to quadratic optimization. J. Optim. Theory Appl. 116(3), 659-678 (2003)

12. Browder, FE: Nonlinear operators and nonlinear equations of evolution in Banach spaces. Proc. Symp. Pure Math. 18 78-81 (1976)

13. Kangtunyakarn, A, Suantai, S: Hybrid iterative scheme for generalized equilibrium problems and fixed point problems of finite family of nonexpansive mappings. Nonlinear Anal. Hybrid Syst. 3, 296-309 (2009)

14. Imnang, S, Suantai, S: Strong convergence theorems for a general system of variational inequality problems, mixed equilibrium problems and fixed points problems with applications. Math. Comput. Model. 52, 1682-1696 (2010)

doi:10.1186/1687-1812-2013-171

Cite this article as: Kangtunyakarn: The methods for variational inequality problems and fixed point of $\kappa$-strictly pseudononspreading mapping. Fixed Point Theory and Applications 2013 2013:171.

\section{Submit your manuscript to a SpringerOpen ${ }^{\circ}$ journal and benefit from:}

- Convenient online submission

- Rigorous peer review

- Immediate publication on acceptance

- Open access: articles freely available online

- High visibility within the field

- Retaining the copyright to your article 